\title{
Ninety-Ninth Annual Report of The American Phytopathological Society
}

\section{REPORT OF THE TREASURER}

The Financial Advisory Committee (FAC) and APS headquarters' staff met on 28 July 2007 in San Diego, CA, to review financial matters related to the operation of the Society and to refine the strategic financial plan. APS continues to make considerable progress in renewing the strategic plan to meet the mission and vision for the future. Council and the officers regularly analyze the external environment and make progress toward assigning responsibility for developing and executing strategies to attain the goals of the strategic plan. Having a strategic financial plan helps us, as a Society, provide a focus for our resources and it is used as a guide for the various committees and staff to do their jobs. Strategic targets help everyone know what is expected from their programs several years in advance so they can build and execute strategies over several budget years. The role of FAC in this process is not to get involved in strategy building, but rather to decide what the financial goals are and to build business plans to meet those goals. FAC continually asks the difficult questions, such as which programs should break even and which are expected to generate surplus to invest in our programs and services that best benefit our members? This plan continues to work for the Society, allowing us to invest in our future.
The FY07 budget was concluded with a surplus of $\$ 295,285$, which represents $6 \%$ of our total income for the period. The 11 income and expense categories for the Society are detailed in Table 1. Our total income $(\$ 4,701,171)$ was derived from the sources as indicated in Figure 1, and our total operating expenses $(\$ 4,405,886)$ incurred during FY07 were partitioned as indicated in Figure 2.

The income and expenses of the Society for the most recent 10 FYs are presented in Table 2. The total assets of the Society as of

TABLE 2. Comparison of The American Phytopathological Society fiscal years 1998 to 2007 after reserve allocation

\begin{tabular}{lccc}
\hline Fiscal year & Income & Expenses & Surplus (deficit) \\
\hline FY07 & $\$ 4,701,171$ & $\$ 4,405,883$ & $\$ 295,288$ \\
FY06 & $\$ 4,289,254$ & $\$ 3,754,227$ & $\$ 535,027$ \\
FY05 & $\$ 3,959,027$ & $\$ 3,819,096$ & $\$ 139,931$ \\
FY04 & $\$ 3,963,006$ & $\$ 3,700,013$ & $\$ 262,993$ \\
FY03 & $\$ 3,748,845$ & $\$ 3,864,217$ & $(\$ 115,372)$ \\
FY02 & $\$ 4,045,587$ & $\$ 3,997,846$ & $\$ 47,741$ \\
FY01 & $\$ 3,952,594$ & $\$ 4,059,101$ & $(\$ 106,507)$ \\
FY00 & $\$ 3,649,863$ & $\$ 3,724,910$ & $(\$ 75,047)$ \\
FY99 & $\$ 3,662,093$ & $\$ 3,692,259$ & $\$ 30,166)$ \\
FY98 & $\$ 3,589,253$ & $\$ 3,431,727$ & $\$ 157,526$ \\
\hline
\end{tabular}

TABLE 1. Audited summary of income and expenses as related to function of The American Phytopathological Society

\begin{tabular}{|c|c|c|c|c|c|c|c|c|c|}
\hline \multirow[b]{2}{*}{ Function } & \multicolumn{4}{|c|}{ Income } & \multicolumn{4}{|c|}{ Expenses } & \multirow[b]{2}{*}{ Profit (loss) } \\
\hline & Actual & $\%$ & Budget & $\%$ & Actual & $\%$ & Budget & $\%$ & \\
\hline Member Services & 324,437 & $7 \%$ & 314,600 & $7 \%$ & 673,756 & $15 \%$ & 767,932 & $18 \%$ & $(349,319)$ \\
\hline Short Courses & 212,375 & $5 \%$ & 89,375 & $2 \%$ & 154,748 & $4 \%$ & 73,568 & $2 \%$ & 57,627 \\
\hline Phytopathology & 847,161 & $18 \%$ & 886,300 & $20 \%$ & 337,912 & $8 \%$ & 376,778 & $9 \%$ & 509,249 \\
\hline Plant Disease & 744,690 & $16 \%$ & 774,000 & $18 \%$ & 348,374 & $8 \%$ & 406,250 & $9 \%$ & 396,316 \\
\hline Phyto News & 10,016 & $0 \%$ & 8,265 & $0 \%$ & 46,468 & $1 \%$ & 63,255 & $1 \%$ & $(36,452)$ \\
\hline MPMI & 591,308 & $13 \%$ & 518,250 & $12 \%$ & 344,958 & $8 \%$ & 368,297 & $9 \%$ & 246,350 \\
\hline PMN & 206,365 & $4 \%$ & 278,248 & $6 \%$ & 269,988 & $6 \%$ & 315,515 & $7 \%$ & $(63,623)$ \\
\hline Online Subscriptions & 30,501 & $1 \%$ & 41,900 & $1 \%$ & 19,967 & $0 \%$ & 32,932 & $1 \%$ & 10,534 \\
\hline APS PRESS & 854,064 & $18 \%$ & 768,998 & $18 \%$ & 627,677 & $14 \%$ & 653,198 & $15 \%$ & 226,387 \\
\hline Annual Meeting & 707,616 & $15 \%$ & 660,195 & $15 \%$ & 580,993 & $13 \%$ & 666,721 & $15 \%$ & 126,623 \\
\hline $\mathrm{G} \& \mathrm{~A}^{\mathrm{a}}$ & 172,638 & $4 \%$ & 7,500 & $0 \%$ & $1,001,045$ & $23 \%$ & 603,167 & $14 \%$ & $(828,407)$ \\
\hline Total & $4,701,171$ & $100 \%$ & $4,347,631$ & $100 \%$ & $4,405,886$ & $100 \%$ & $4,327,613$ & $100 \%$ & 295,285 \\
\hline Surplus (loss) & & & & & & & & & 295,285 \\
\hline
\end{tabular}

${ }^{\mathrm{a}} \mathrm{G} \& \mathrm{~A}$ is assigned to functions based on payroll dollars.

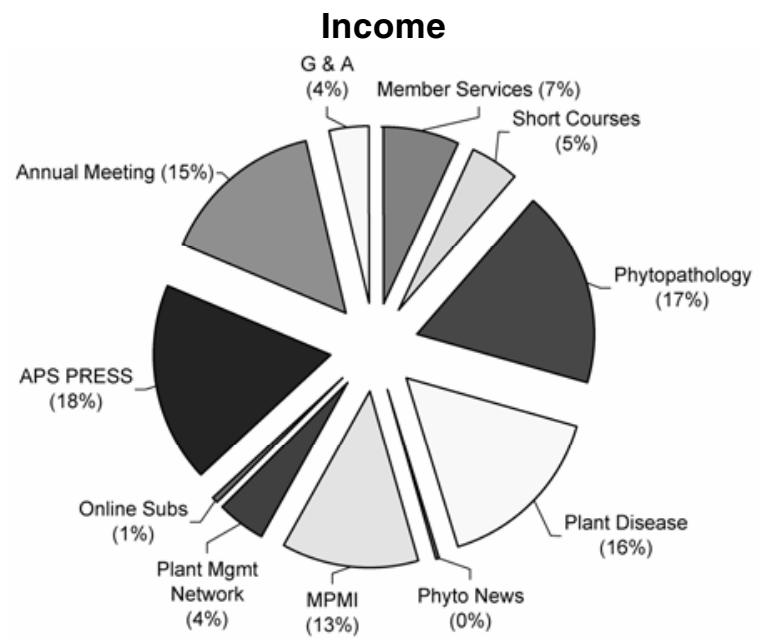

Fig. 1.

doi:10.1094/PHYTO-98-1-0017 
30 June 2007, including restricted funds, were $\$ 7.8$ million and current liabilities totaled $\$ 2.6$ million. This resulted in total net assets of $\$ 5.2$ million.

The positive net income from programs and investments continue to be re-invested in the Society initiatives that continue to enhance member services, including APSnet, our Washington, DC presence (Eversole Associates), the increased use of reserves of the Society in supporting programming at the APS annual meeting, and investing in the Plant Management Network. The future of the Plant Management Network continues to look promising with increased content in four journals sponsored in part by more than 60 partners, while our presence in Washington continues to bring support to our discipline and Society. The Treasurer can be contacted at Rowe.4@OSU.edu.

\section{REPORT OF THE AUDITOR}

The American Phytopathological Society has its accounts audited annually by a certified public accountant. In recent years, the accounting firm has been Lethert, Skwira, Schultz, \& Co., St. Paul, MN. Only the Balance Sheet and the Statement of Changes in Net Assets, as of 30 June 2007, are being published in detail. Any member wishing a copy of the full audit may obtain one on request.
The American Phytopathological Society Statement of Changes in Net Assets Year ended 30 June 2007 (with comparative totals for 2006)

\begin{tabular}{|c|c|c|}
\hline & 2007 & 2006 \\
\hline \multicolumn{3}{|l|}{ Unrestricted net assets (support and revenue) } \\
\hline Federal financial assistance & 28,699 & 44,051 \\
\hline Contributions & 98,626 & 75,950 \\
\hline Membership dues & 284,078 & 282,910 \\
\hline Subscriptions & $1,867,109$ & $1,847,557$ \\
\hline Reprints and demand articles & 61,045 & 67,144 \\
\hline Abstracts & 7,020 & 8,575 \\
\hline Page charges & 204,860 & 219,014 \\
\hline Processing fees & 114,920 & 116,210 \\
\hline Back issues & 10,749 & 8,278 \\
\hline Color charges & 142,771 & 118,803 \\
\hline Advertising & 45,896 & 47,417 \\
\hline Books, slides, CD-Roms, and clothing & 848,359 & 838,016 \\
\hline Annual meeting & 707,616 & 533,267 \\
\hline Short courses & 43,575 & 39,825 \\
\hline Royalty income & 9,182 & 27,491 \\
\hline Investment income (note 4 ) & 176,847 & 87,937 \\
\hline Gain on sale of investments (note 4) & 10,473 & 189,491 \\
\hline Unrealized gain (loss) on investments (note 4) & 331,668 & $(112,682)$ \\
\hline Other & 16,692 & 20,974 \\
\hline Total support and revenue & $5,010,185$ & $4,460,228$ \\
\hline \multicolumn{3}{|l|}{ Net assets released from restrictions } \\
\hline Restrictions satisfied by payments & 87,347 & 65,799 \\
\hline \multicolumn{3}{|l|}{ Expenses } \\
\hline \multicolumn{3}{|l|}{ Program services } \\
\hline Member services & 700,938 & 688,594 \\
\hline Journals & $1,347,698$ & $1,226,883$ \\
\hline Books & 642,990 & 670,697 \\
\hline Annual meeting & 646,874 & 413,024 \\
\hline Grants and awards & 42,000 & 29,970 \\
\hline Short courses and workshops & 107,237 & 98,002 \\
\hline Online services & 19,967 & 18,314 \\
\hline Total program services & $3,507,704$ & $3,145,484$ \\
\hline \multicolumn{3}{|l|}{ Supporting services } \\
\hline Management and general & 987,126 & 730,690 \\
\hline Fundraising & 4,077 & 751 \\
\hline Total supporting services & 991,203 & 731,441 \\
\hline Total expenses & $4,498,907$ & $3,876,925$ \\
\hline Increase in unrestricted net assets & 598,625 & 649,102 \\
\hline \multicolumn{3}{|l|}{ Temporarily restricted net assets } \\
\hline Support for books & 12,689 & 30,119 \\
\hline Support for annual meeting socials & 25,040 & 145,689 \\
\hline Support for student travel & 6,052 & 5,216 \\
\hline Investment income (note 4$)$ & 42,735 & 22,572 \\
\hline Gain on sale of investments (note 4 ) & 2,352 & 77,198 \\
\hline Unrealized gain (loss) on investments (note 4) & 85,947 & $(43,592)$ \\
\hline Restrictions satisfied by payments & $(87,347)$ & $(65,799)$ \\
\hline Increase in temporarily restricted net assets & 87,468 & 171,403 \\
\hline \multicolumn{3}{|l|}{ Permanently restricted net assets } \\
\hline Support for operations 0 & 34,314 & 4,730 \\
\hline Support for student travel & 38,259 & 25,698 \\
\hline Support for student speakers & 270 & 235 \\
\hline Support for research and education & 1,195 & 1,243 \\
\hline Support for international travel & 200 & 480 \\
\hline Unrealized gain (loss) on investments (note 4) & 164 & $(821)$ \\
\hline Increase in permanently restricted net assets & 74,402 & 31,565 \\
\hline Increase in net assets & 760,495 & 852,070 \\
\hline Net assets, beginning of year & $4,857,859$ & $4,005,789$ \\
\hline Change in unfunded pension liability (note 12 ) & $(41,773)$ & - \\
\hline Net assets, end of year & $5,576,581$ & $4,857,859$ \\
\hline
\end{tabular}
67,144 8,575 210 8,278 838,016 33,267 491 (2,682) 0,228
The American Phytopathological Society Balance Sheet Year ended 30 June 2007 (with comparative totals for 2006)

\begin{tabular}{|c|c|c|}
\hline & 2007 & 2006 \\
\hline \multicolumn{3}{|l|}{ Assets } \\
\hline Cash (note 3$)$ & $1,239,172$ & $1,008,526$ \\
\hline Accounts receivable, net (notes 2 and 15) & 407,554 & 479,109 \\
\hline Unconditional promises to give & 53,412 & 69,879 \\
\hline Investments (note 4) & $4,753,607$ & $4,067,542$ \\
\hline Prepaid expenses (note 5) & 387,485 & 442,036 \\
\hline Inventory & 633,688 & 619,517 \\
\hline Prepaid pension (note 12 ) & 60,702 & 47,617 \\
\hline Property and equipment, net (note 6) & 409,809 & 360,632 \\
\hline Total assets & $7,945,429$ & $7,094,858$ \\
\hline \multicolumn{3}{|l|}{ Liabilities and net assets } \\
\hline Accounts payable (note 15 ) & 320,387 & 253,466 \\
\hline Accrued payroll and payroll taxes & 43,930 & 40,757 \\
\hline Accrued pension expense & 81,144 & 81,844 \\
\hline Agency funds payable & 15,341 & 13,442 \\
\hline Deferred revenues (note 7) & $1,866,273$ & $1,847,490$ \\
\hline Underfunded pension plan liability (note 12 ) & 41,773 & - \\
\hline Total liabilities & $2,368,848$ & $2,236,999$ \\
\hline \multicolumn{3}{|l|}{ Net assets } \\
\hline Unrestricted (note 8) & $4,052,703$ & $3,495,852$ \\
\hline Temporarily restricted (note 9) & 408,770 & 321,301 \\
\hline Permanently restricted (note 10 ) & $1,115,108$ & $1,040,706$ \\
\hline Total net assets & $5,576,581$ & $4,857,859$ \\
\hline Total liabilities and net assets & $\$ 7,945,429$ & $7,094,858$ \\
\hline
\end{tabular}

政

